\title{
New mid-cervical vertebral morphotype of Spinosauridae from the Kem Kem Group of Morocco
}

\author{
Bradley D. McFeeters \\ Department of Earth Sciences, Carleton University, 1125 Colonel By Drive, Ottawa, Ontario, Canada, K1S 5B6 \\ bradleymcfeeters@cmail.carleton.ca
}

\begin{abstract}
An unusual mid-cervical vertebra belonging to a large spinosaurid from the Cenomanian Kem Kem Group of Morocco is described. It is compared to the characteristic morphology of each reconstructed cervical position in Spinosaurus aegyptiacus, based on a recent composite reconstruction that incorporates most previously referred material from this unit. Rather than conforming to any of the previously identified cervical positions in its morphology, the specimen displays a unique combination of mid-cervical characters, with the relatively anteroposteriorly short centrum suggesting a position as $\mathrm{C} 4$, and the form of the neural arch laminae suggesting a position as $\mathrm{C} 5$ or C6. Furthermore, it displays two characters that are previously unknown in spinosaurid mid-cervicals from the Kem Kem Group: a rounded hypapophyseal tuberosity that is not continuous with a ventral keel, and a moderately developed, dorsally oriented epipophysis that does not overhang the postzygapophysis posteriorly. The diagnostic value of positionally variable cervical vertebral characters in spinosaurid systematics is discussed. Although limited, the new data could lend support to the contentious hypothesis that two spinosaurid taxa are represented in the Kem Kem Group.
\end{abstract}

\section{INTRODUCTION}

Spinosauridae is a clade of large, specialized tetanuran theropods that was widely distributed during the Cretaceous (Hone and Holtz 2017). Recent years have seen intense scientific interest in spinosaurid fossils from the Cenomanian (Upper Cretaceous) Kem Kem Group of eastern Morocco (Ibrahim et al. 2020a), with new material fuelling numerous investigations into the morphology and palaeobiology of these spectacular giant theropods (Ibrahim et al. 2014; Evers et al. 2015; Hendrickx et al. 2016; Gimsa et al. 2016; Henderson 2018; Maganuco and Dal Sasso 2018; Arden et al. 2019; Lakin and Longrich 2019; Heckeberg and Rauhut 2020; Ibrahim et al. 2020b; Smyth et al. 2020; Beevor et al. 2021; Hone and Holtz in press). Most of the known spinosaurid material from the Kem Kem Group consists of isolated elements, often collected without precise geological context, although one associated partial skeleton was recently described (Ibrahim et al. 2014; Ibrahim et al. 2020b). In the absence of more complete remains, uncertainty has persisted surrounding the precise axial positions of some of the isolated vertebral elements, as well as the number of distinct spinosaurid taxa represented in this sample.
Fragmentary spinosaurid craniodental material from the Kem Kem Group was initially referred to Spinosaurus cf. S. aegyptiacus (Buffetaut 1989), indicating affinities with the type species originally described by Stromer (1915) on the basis of a partial skeleton, now destroyed, from the Bahariya Formation of Egypt. Russell (1996) described numerous isolated spinosaurid vertebrae from the Kem Kem Group as belonging to two new taxa, Spinosaurus maroccanus and Sigilmassasaurus brevicollis, although the latter was not yet recognized as a spinosaurid at that time. Both of these taxa were subsequently proposed to be junior synonyms of Spinosaurus aegyptiacus (Sereno et al. 1998; Ibrahim et al. 2014, 2020a), resulting in only a single spinosaurid taxon being recognized in this assemblage. Other recent studies, however, have continued to recognize at least two distinct spinosaurid taxa in the Kem Kem Group (Evers et al. 2015; Hendrickx et al. 2016; Hone and Holtz 2017; Arden et al. 2019; Lakin \& Longrich 2019), although these are not necessarily the same two taxa proposed by Russell (1996). Evers et al. (2015), for example, synonymized Spinosaurus maroccanus with Sigilmassasaurus brevicollis, but referred other vertebral material from the Kem Kem Group to a second, indeterminate spinosaurid species. 
McFeeters - Vertebral morphotype of Spinosauridae

Most recently, Smyth et al. (2020) reevaluated the taxonomy of the Kem Kem Group spinosaurids and concluded that the recognition of only a single taxon, Spinosaurus aegyptiacus, was justified. They also produced a revised reconstruction of the post-atlas cervical and anterior dorsal vertebral series of Spinosaurus aegyptiacus, with the known fossil material representing an essentially complete sampling of axial positions C2-D3 (second cervical to third dorsal) in this taxon. Previously identified vertebral characters supporting the presence of multiple spinosaurid taxa in the Kem Kem Group were reinterpreted as resulting from individual variation, taphonomic damage, or variation in the axial position of the elements (Smyth et al. 2020). Here, I describe a cervical vertebra of a spinosaurid from the Kem Kem Group exhibiting an unusual combination of positionally variable characters. This specimen offers further opportunity to test the proposed characterizations of individual axial positions that make up the composite reconstructed neck of Spinosaurus aegyptiacus (Smyth et al. 2020), and the hypothesis that all spinosaurid material from the Kem Kem Group is referable to a single species.

\section{MATERIALS AND METHODS}

The specimen, ROM 65537 (Figs. 1-3; Table 1), consists of a nearly complete isolated vertebra. It was briefly mentioned as spinosaurid by Evers et al. (2015), but has not been previously figured or comprehensively described. Unfortunately, as is often the case with commercially collected Kem Kem Group material acquired by museum collections (Russell 1996; Ibrahim et al. 2020a), further information on the geological context of this specimen is not available. There are no apparent signs of restoration or alteration, apart from the right prezygapophysis (K. Seymour, pers. comm. November 2020). It is readily identifiable as a postaxial cervical, based on a combination of characters including large, elevated, dorsomedially facing prezygapophyses; slight ventral deflection of the anterior articular surface; parapophyses positioned low on the centrum; and ventrally projecting diapophyses. In practically all of its individual morphological characters, ROM 65537 closely resembles previously described spinosaurid cervicals from the Kem Kem Group (Russell 1996; Evers et al. 2015; Lakin and Longrich 2019; Smyth et al. 2020). In particular, the combination of a relatively elongated centrum, modest neural arch lamination, and a camerate grade of pneumatization with a single pair of central pneumatic foramina (Benson et al. 2012) support the referral of ROM 65537 to Spinosauridae, while distinguishing it from other large theropods (e.g., Carcharodontosauridae, Abelisauroidea) known to occur in this unit (Chiarenza and Cau 2016; Ibrahim et al. 2020a).
Material examined first hand by the author includes spinosaurid cervicals in the collections of the Canadian Museum of Nature and the Royal Ontario Museum. The angles of the anterior articular surface and the divergence of the prezygapophyses were measured from photographs. Other data were sourced from the literature. Terminology for vertebral laminae and fossae follows Wilson (1999) and Wilson et al. (2011).

\section{Institutional abbreviations: BSPG, Bayerische}

Staatssammlung für Paläontologie und Geologie, Munich, Germany; CMN, Canadian Museum of Nature, Ottawa, ON, Canada; FSAC, Faculté des Sciences Aïn Chock (University of Casablanca), Casablanca, Morocco; MPDM, Musée Parc des Dinosaures, Mèze, France; MSM, Museo Paleontológico Juan Cano Forner, Sant Mateu, Spain; NHMUK, Natural History Museum, London, UK; ROM, Royal Ontario Museum, Toronto, ON, Canada.

\section{DESCRIPTION OF ROM 65537}

Centrum: The centrum is strongly opisthocoelous (Figs. 1, 2 ). It is moderately elongate, with a length/posterior width ratio of 1.65, and a length/posterior height ratio of 1.86 . The hemispherical anterior articular surface is wider than tall and is deflected slightly ventrally from the long axis of the centrum, at an angle of approximately $15^{\circ}$. In anterior view, the dorsal margin of the anterior articular surface appears to have a slight medial indentation for the floor of the neural canal, though the anterior convexity is damaged in this region. There is no central anterior tubercle. A flattened rim surrounds the anterior convexity laterally and dorsally. In ventral view, a prominent transverse groove separates the anterior articular surface from the remainder of the centrum. The ventral surface of the centrum immediately posterior to this groove has a rugose, faintly ridged texture.

The parapophyses are anteroposteriorly elongate and are positioned lateroventrally on the centrum. The anterior end of the left parapophysis meets the posterior rim of the anterior articular surface, whereas the right parapophysis is fully separated from the anterior articular surface by a continuation of the transverse groove, possibly due to damage. The left parapophysis is broken lateroventrally, revealing a camerate grade of pneumatization. The prominent ventral posterior parapophyseal ridges extend posteromedially from the parapophyses and contribute to the sharply waisted outline of the centrum with non-parallel sides in ventral view. Positioned between the parapophyses on the ventral midline of the centrum is a broad, rounded, deeply rugose hypapophyseal tuberosity, which extends approximately as far posteriorly as the maximum lateral constriction of the centrum (Fig. 3A). There is no midline ridge or keel posterior to the hypapophyseal tuberosity. 


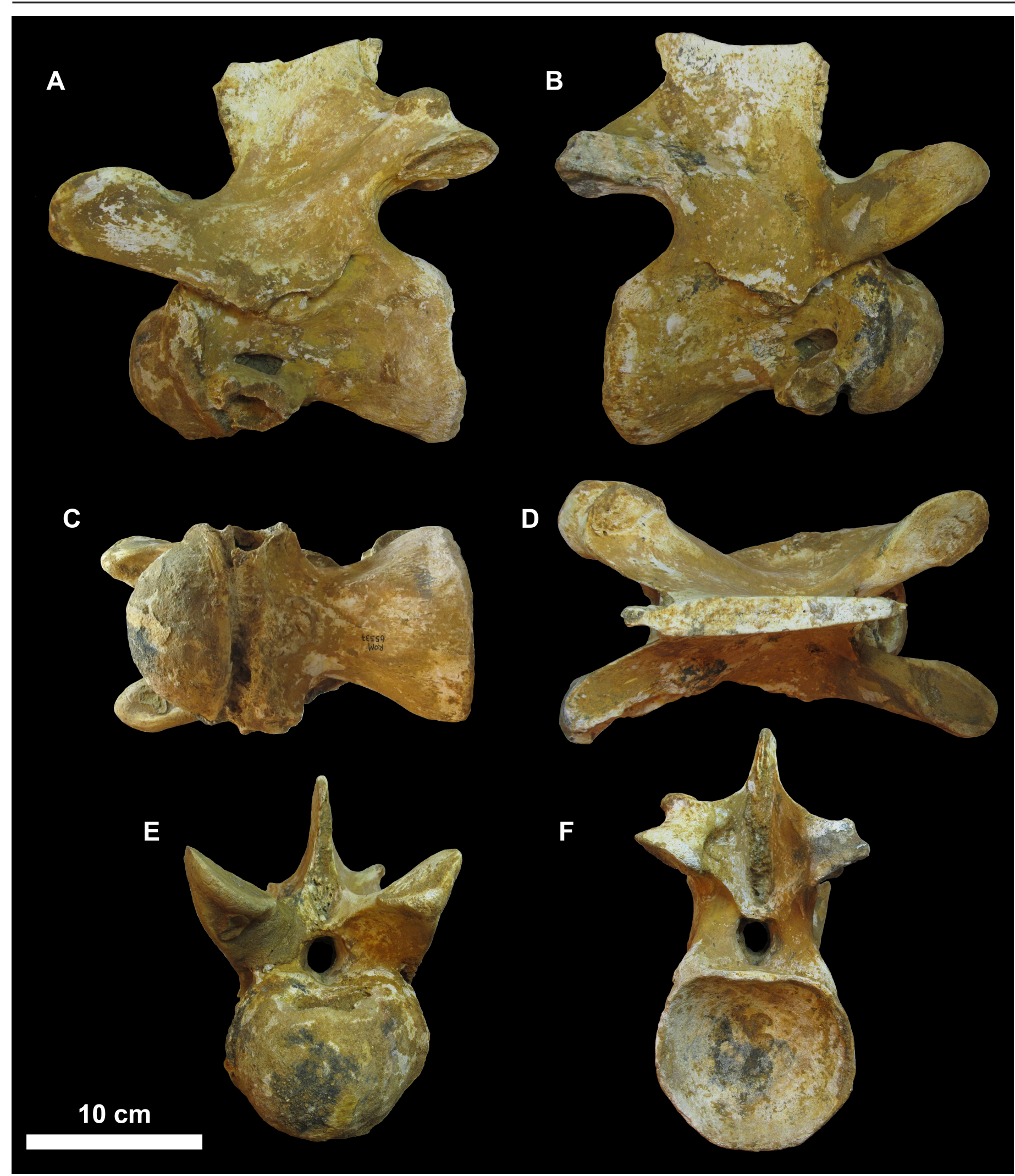

Figure 1. ROM 65537, mid-cervical vertebra of Spinosauridae indet., in A, left lateral view, scaled to centrum length; B, right lateral view, scaled to centrum length; $C$, ventral view, scaled to centrum length; $D$, dorsal view, scaled to neural arch length; E, anterior view, scaled to centrum width; and F, posterior view, scaled to centrum width. Refer to Figure 2 for labels of anatomical features. 

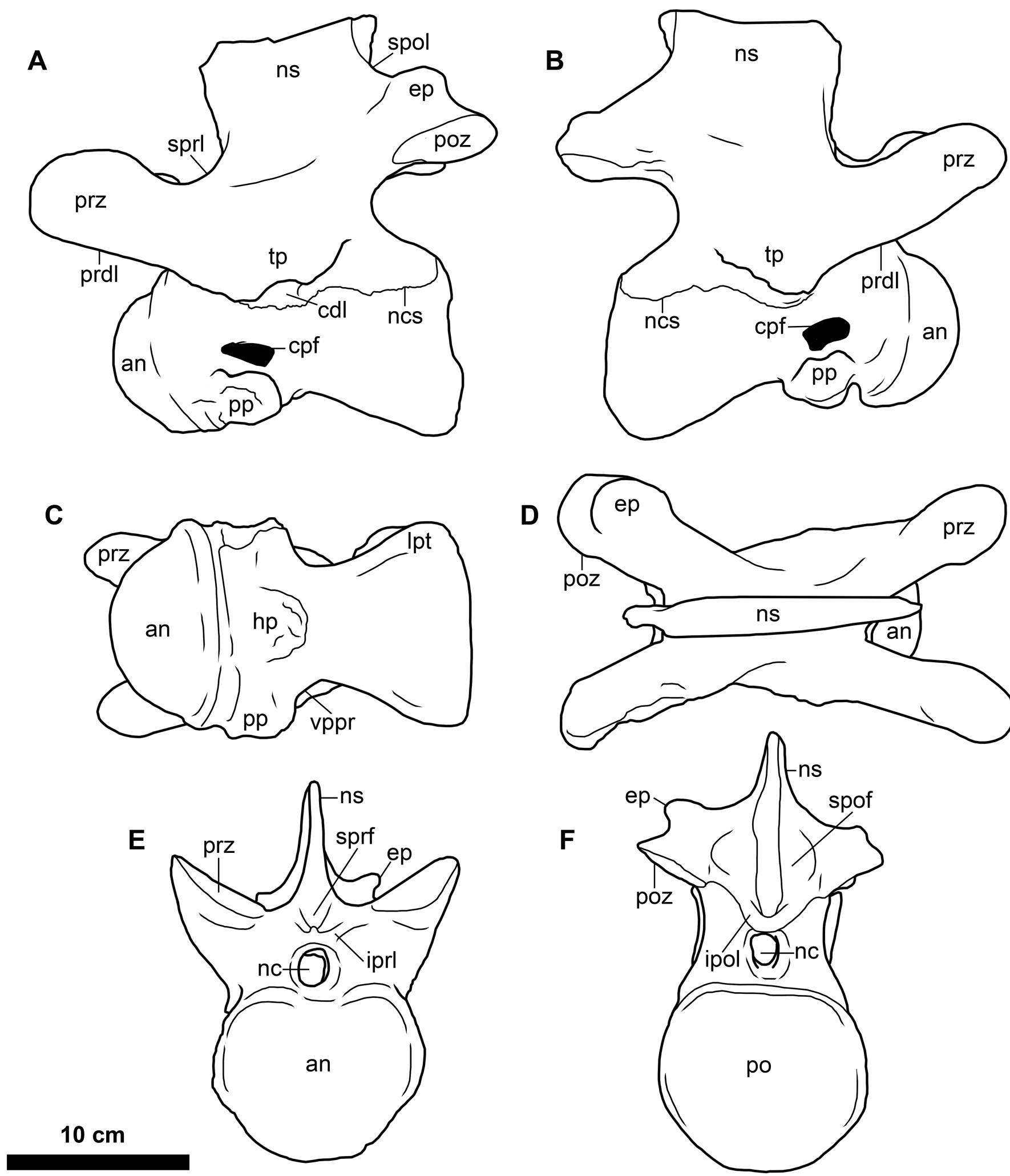

Figure 2. Interpretive drawings of ROM 65537, mid-cervical vertebra of Spinosauridae indet., in A, left lateral view; B, right lateral view; C, ventral view; D, dorsal view; E, anterior view; and F, posterior view. Abbreviations: an, anterior articular surface; cdl, centrodiapophyseal lamina; cpf, central pneumatic foramen; ep, epipophysis; hp, hypapophyseal tuberosity; ipol, interpostzygapophyseal lamina; iprl, interprezygapophyseal lamina; Ipt, laterally placed tubercle; nc, neural canal; ncs, neurocentral suture; ns, neural spine; po, posterior articular surface; pp, parapophysis; prdl, prezygodiapophyseal lamina; prz, prezygapophysis; spof, spinopostzygapophyseal fossa; spol, spinopostzygapophyseal lamina; sprf, spinoprezygapophyseal fossa; sprl, spinoprezygapophyseal lamina; tp, transverse process; vppr, ventral posterior parapophyseal ridge. 


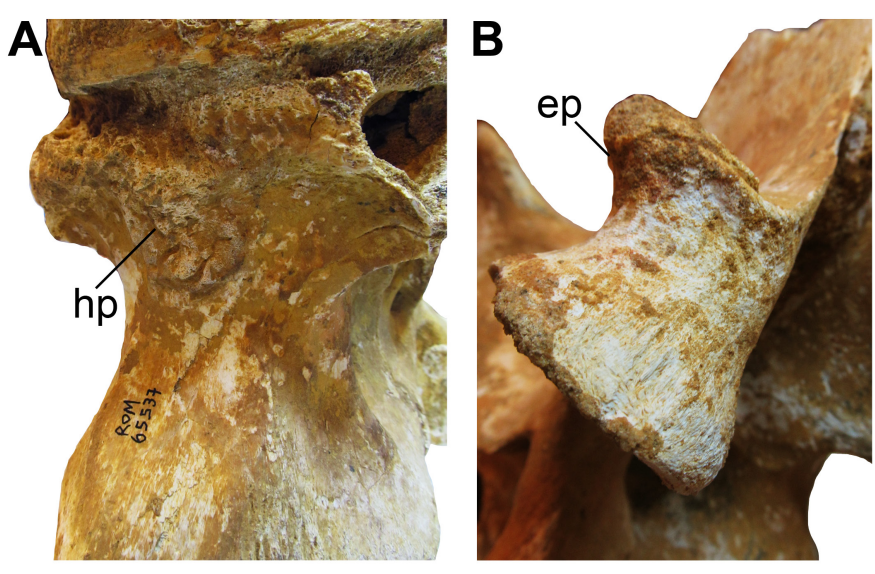

Figure 3. Unique features of ROM 65537, mid-cervical vertebra of Spinosauridae indet.: A, hypapophyseal tuberosity in oblique left ventrolateral view (anterior end of centrum towards top of page); B, upright epipophysis in oblique left posterolateral view. Abbreviations as in Figure 2. Refer to Figures $1 \& 2$ for cardinal views and scale bars.

The ventral edge of the centrum posterior to the parapophyses is angled posteroventrally in lateral view. There is no horizontal posteroventral triangular platform, but laterally placed tubercles are present (Evers et al. 2015). Immediately anterior to the posterior articular surface, the lateral and ventral surfaces of the centrum are finely textured with a series of faint anteroposterior ridges. The posterior articular surface is subcircular in outline, slightly wider than high, with a flat, straight dorsal edge that is slightly damaged. There is a single pneumatic foramen on either side of the centrum, with its long axis oriented anteroposteriorly. The anterior end of the left central pneumatic foramen is slightly tapered, whereas the anterior margin of the right central pneumatic foramen is deeper and rounded. On both sides, the central pneumatic foramen lies directly dorsal to the parapophysis, with its posterior margin not projecting past the posterior extent of the parapophysis.

Neural arch: The large prezygapophyses project farther anteriorly than the anterior articular surface of the centrum. The prezygapophyseal facets face dorsomedially, with their long axes oriented anterolateral to posteromedial. In dorsal view, the prezygapophyses diverge laterally at an angle of approximately $60^{\circ}$. The surfaces of the right prezygapophysis and its pedicel are largely reconstructed (darker areas in Figure 1). There is no centroprezygapophyseal fossa on the left pedicel.

Stout interprezygapophyseal laminae separate the neural canal from the spinoprezygapophyseal fossa. A low, midline ridge divides the floor of the spinoprezygapophyseal fossa anterior to the neural spine. The large neural spine is broken dorsally. Its anterior margin is rugose, and leans slightly anterodorsally. The spinoprezygapophyseal lamina
Table 1. Measurements of ROM 65537

Dimension

Measurement (mm)

Centrum length 195

Centrum length (excl. anterior hemispherical surface) 144

Anterior articular surface height (midline)

Anterior articular surface height (maximum)

Anterior articular surface width

Anterior articular surface width (excl. flattened rim)

Posterior articular surface height

Posterior articular surface width

Width across prezygapophyses

Width across postzygapophyses

Neural spine height

Neural spine mediolateral thickness (maximum)

Total height

* Incompletely preserved

joins the neural spine slightly posterior to the neural spine's anterior margin. The neural spine is mediolaterally compressed, with its thickness gradually increasing posteriorly for the anterior half of its length. Posterior to the spinopostzygapophyseal laminae, the posterior strip of the neural spine is abruptly reduced in thickness. The posterior edge of the neural spine is separated from the neural canal by the interpostzygapophyseal laminae, which project posteriorly beyond the edge of the neural spine and define the floor of the spinopostzygapophyseal fossa.

The base of the transverse process is anteroposteriorly extensive. Its anterior part is continuous with the prezygodiapophyseal lamina, which has a straight ventral edge that covers the anterior margin of the neural arch pedicels in lateral view. The posterior lamina of the transverse process terminates near the posterior end of the neural arch pedicels. Two foramina or deep fossae, subequal in size, enter the neural arch one anterior and one posterior to the diapophysis, but are not visible in lateral view due to the overhanging laminae. The diapophysis is not completely preserved on either side, but projects ventrally. It is triangular in cross-section, with a flat lateral surface. The midpoint of the diapophysis is positioned more posteriorly than the midpoint of the parapophysis. The diapophysis is connected to the centrum by a thick, undivided centrodiapophyseal lamina. A suture line is visible between the neural arch and the centrum.

The postzygapophysis and epipophysis are damaged on the right side of the specimen, so the description will focus on the better-preserved left side. When complete, the postzygapophyses would have spanned a similar width as the prezygapophyses. The comma-shaped postzygapophyseal facet is directed ventrolaterally. The low, blunt epipophysis 
McFeeters - Vertebral morphotype of Spinosauridae

projects dorsolaterally (Fig. 3B). The anterior edge of the epipophysis forms a lamina directed towards the prezygapophysis, but is not continuously joined to it across the lateral surface of the neural arch. The posterior edge of the epipophysis is approximately vertical, so the epipophysis does not overhang the postzygapophysis posteriorly. There is a relatively long, sloping dorsal surface of the postzygapophysis between the base of the epipophysis and the posterior margin of the postzygapophyseal facet.
COMPARISONS TO SPECIMENS REFERRED TO SPINOSAURUS

The following comparisons refer to the cervical morphotypes identified in the composite reconstruction of Spinosaurus aegyptiacus, as determined by Smyth et al. (2020:tab. 1), including material identified by Evers et al. (2015) as Sigilmassasaurus brevicollis (BSPG 2006 I 53, CMN 50791, NHMUK PV R 16427) and Spinosauridae
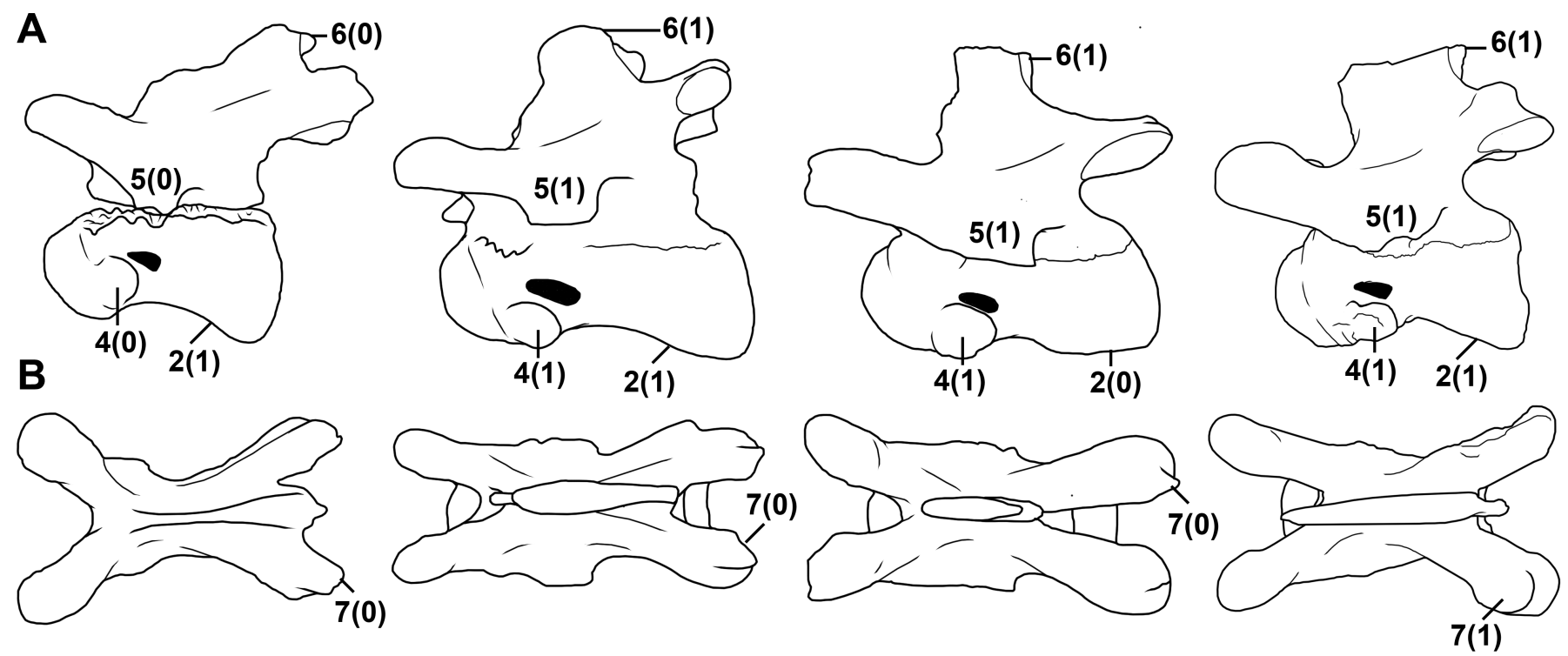

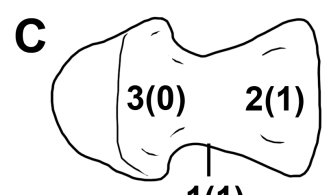

1(1)

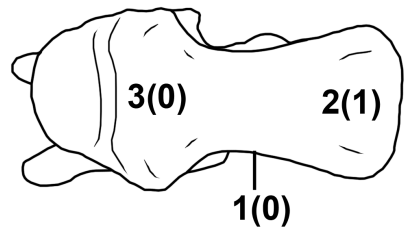

"C5"

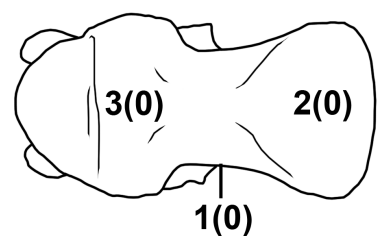

"C6"

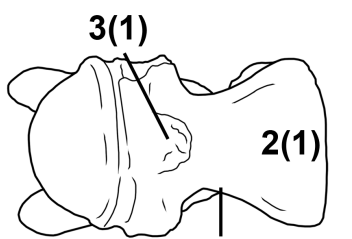

1(1)

ROM 65537

Figure 4. Comparison of spinosaurid mid-cervical morphotypes from the Kem Kem Group in A, left lateral; B, dorsal; and C, ventral views; drawn to the same approximate centrum height. 'C4' is represented by the centrum of FSAC-KK-5017 (after Smyth et al. 2020:fig. 1) and the neural arch of NHMUK PV R 16427 (after Evers et al. 2015:fig. 4). The composite illustration is justified in that both specimens can be referred to the same position as NHMUK PV R 16429 (Evers et al. 2015:fig. 18; Smyth et al. 2020:tab. 1), in which the centrum and the neural arch are both present but incomplete. ' ${ }^{5}$ ' is represented by FSACKK-5019 (after Smyth et al. 2020:fig. 2). 'C6' is represented by CMN 50791 (after Evers et al. 2015:fig. 1). Variation in the following characters is labeled, with the arbitrary convention of state (1) corresponding to the condition in ROM 65537: 1) Centrum shape: anteroposteriorly longer with a subparallel-sided mid-section in ventral view (0) vs. anteroposteriorly shorter with a sharply waisted mid-section in ventral view (1). 2) Posteroventral end of centrum in mature individuals: flat ventral triangular platform (0) vs. laterally placed tubercles (1). 3) Ventral region of centrum between parapophyses: hyapophyseal tuberosity absent (0) vs. present (1). 4) Position of parapophysis on centrum: lateral (0) vs. ventrolateral, influencing the ventral outline of the centrum in lateral view (1). 5) Transverse process of neural arch: anteroposteriorly narrower (0) vs. anteroposteriorly wider (1). 6) Neural spine, posterior extent: considerably overlaps postzygapophyseal facet in lateral view (0) vs. does not overlap postzygapophyseal facet in lateral view (1). 7) Epipophysis orientation: directed posterodorsally, overhanging the posterior margin of the postzygapophysis in dorsal view (0) vs. oriented laterodorsally, widely separated from posterior margin of postzygapophysis in dorsal view (1). 
indet. (CMN 41768, CMN 50790, NHMUK PV R

16429). Except where specifically noted, anatomical comparisons refer generally to the sample of Kem Kem Group spinosaurid cervicals previously described by Evers et al. (2015), and Smyth et al. (2020). The comparisons to other mid-cervical vertebrae (C4-C6) are summarized in Figure 4. Smyth et al. (2020:tab. 1) considered C3 of Spinosaurus to be known from only a single specimen, "Halswirbel a" of BSPG 1912 VIII 19, the destroyed holotype of Spinosaurus aegyptiacus from the Bahariya Formation (Stromer 1915, Evers et al. 2015). However, a second described specimen could also belong to this position. The laterally flaring spinopostzygapophyseal laminae and correspondingly very high spinopostzygapophyseal fossa, previously cited to support the identification of "Halswirbel a" as C3 (Evers et al. 2015), are also observed in CMN 41768, a specimen from the Kem Kem Group described by Russell (1996:fig 4D) as a paratype of Spinosaurus maroccanus. Smyth et al. (2020:tab. 1) listed CMN 41768 as representing C5, the same position as another Spinosaurus maroccanus paratype, CMN 50790; however, its posterior profile is markedly different from that specimen (Fig. 5), as well as from vertebrae assigned to C4 (eg. NHMUK PV R 16427, Evers et al. 2015:fig. 4). CMN 41768 is tentatively reidentified here as also representing C3, agreeing with the original interpretation by Russell (1996) that it represents a more anterior position than CMN 50790.

The C3 vertebrae differ from ROM 65537 in having smaller, less anterodorsally expanded prezygapophyses (extending scarcely beyond the anterior end of the centrum in CMN 41768); an anteroposteriorly narrow transverse process restricted to the anterior half of the neural arch; a posteriorly expansive neural spine that overlies the postzygapophysis in lateral view; a dorsolaterally more extensive spinopostzygapophyseal region; and large, posteriorly or posterodorsally oriented epipophyses that overhang the posterior end of the postzygapophysis, with the posteroventral base of the epipophysis close to the posterior margin of the postzygapophyseal facet. The centrum of CMN 41768 is poorly preserved, but appears to be less strongly waisted in lateral and ventral views compared to ROM 65537.

Vertebrae identified by Smyth et al. (2020) as C4 agree with ROM 65537 in having a relatively compact (compared to more posterior mid-cervical positions) and distinctively waisted centrum, with little or no length of the lateral sides of the centrum being parallel posterior to the parapophyses in ventral view (e.g., NHMUK PV R 16429, Evers et al. 2015:fig. 18C; FSAC-KK-5017, Smyth et al. 2020:fig. 1B). The ratios of centrum length to posterior width and height in ROM 65537 are consistent with identified C4 specimens, being between those of FSAC-KK-5017 and FSACKK-5018 (Smyth et al. 2020). Specimens identified as C4

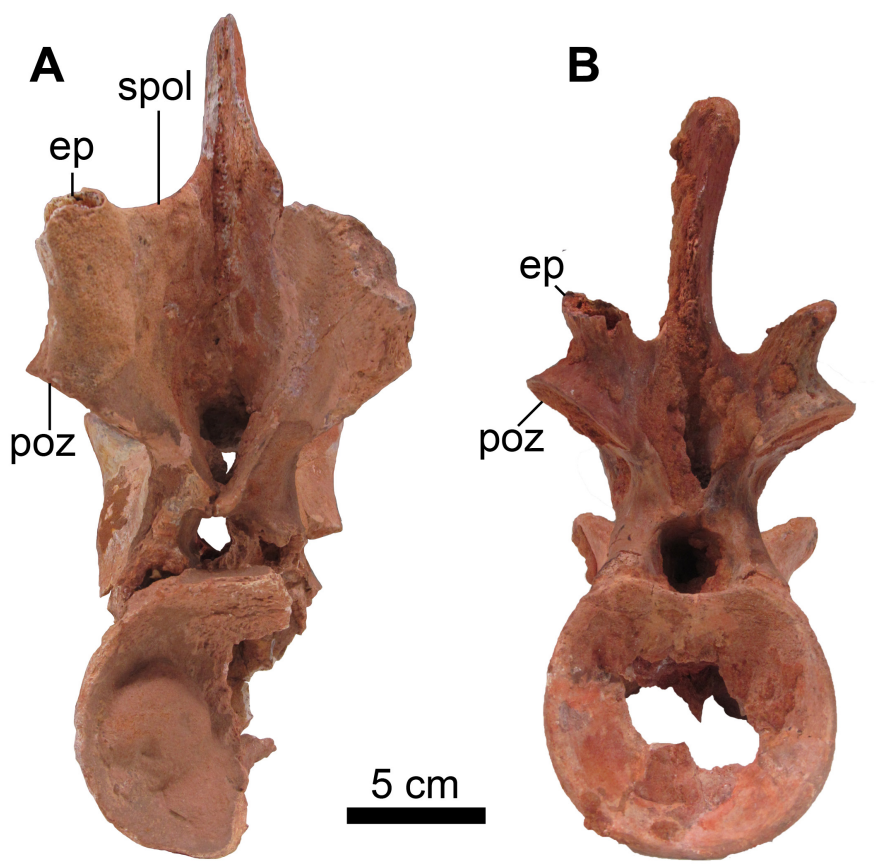

Figure 5. Comparison of cervical vertebrae referred to 'Spinosaurus maroccanus' (Russell 1996) in posterior view: A, CMN 41768 (C3?); B, CMN 50790 (C5). Abbreviations: ep, epipophysis; poz, postzygapophysis; spol, spinopostzygapophyseal lamina.

also possess laterally placed tubercles and lack a keel on the ventral side of the centrum, but differ from ROM 65537 in lacking the rounded hypapophyseal tuberosity. In NHMUK PV R 16429, the anterior region of the ventral surface has a potentially homologous, but comparatively narrow, slightly raised surface (Evers et al. 2015:fig 18C), whereas in FSAC-KK-5017 there is no raised structure but the anterior ventral surface is very rugose (Smyth et al. 2020:fig. 1E). The parapophyses of vertebrae identified as $\mathrm{C} 4$ are less ventrally positioned than in ROM 65537, not contributing to the ventral outline of the centrum in lateral view (Smyth et al. 2020:fig. 1), especially in "Halswirbel b" of BSPG 1912 VIII 19 , where they are positioned essentially at the mid-height of the centrum (Stromer 1915). The position of the central pneumatic foramen in these vertebrae is also posterodorsal to the parapophysis, rather than directly dorsal to it as in ROM 65537. The importance of this difference is questionable, however, given the extensive variation in pneumatic openings that has been documented in theropod vertebrae, even between opposite sides of the same element (Evers et al. 2015). On the neural arch, the $\mathrm{C} 4$ vertebrae differ from ROM 65537 in having a somewhat greater, though variable, divergence angle between the prezygapophyses in dorsal view (NHMUK PV R 16427, Evers et al. 2015:fig. 4C); a relatively high ventral margin of the prezygodiapophysial lamina that exposes a considerable amount of the anterior margin of the neural arch pedicel in lateral view (NMNUK 
McFeeters - Vertebral morphotype of Spinosauridae

PV R 16427, Evers et al. 2015:fig. 4E-5; NHMUK PV R 16429, Evers et al. 2015:fig. 18A); an anteroposteriorly narrower transverse process; and posteriorly oriented epipophyses that overhang the posterior end of the postzygapophysis, with the posteroventral base of the epipophysis close to the posterior margin of the postzygapophyseal facet (NMNUK PV R 16427, Evers et al. 2015:fig. 4A; Smyth et al. 2020). A spinosaurid neural arch assigned to C4 from the Kem Kem Group, NHMUK PV R 16427, also differs from ROM 65537 in the more posterior expansion of the neural spine partially overlying the postzygapophyseal facets in lateral view (Evers et al. 2015:fig. 4E, F), as in more anterior cervicals (BSPG 1912 VIII 19 "Halswirbel a"; CMN 41768); however, "Halswirbel b" of BSPG 1912 VIII 19 from the Bahariya Formation does not differ from ROM 65537 in this regard (Stromer 1915, Smyth et al. 2020:fig. 9B). Vertebrae identified by Smyth et al. (2020) as C5 agree with ROM 65537 in the absence of a ventral keel and the presence of laterally placed tubercles, but are typically much more elongate, with a substantial nearly parallel-sided section of the mid-centrum in ventral view, and a total centrum length equal to at least twice the posterior width (Smyth et al. 2020). Although the parapophysis contributes to the ventral outline of typical C5 vertebrae in lateral view (e.g., FSAC-KK-5019, Smyth et al. 2020:fig. 2), the ventral posterior parapophyseal ridge is less strongly inflected from the posterior part of the centrum than in ROM 65537. The centra of the C5 vertebrae also differ from ROM 65537 in having no hypapophyseal tuberosity, and when its position is determinable (e.g., FSAC-KK-5019), the central pneumatic foramen is positioned posterodorsal rather than dorsal to the parapophysis. On the neural arch, the C5 vertebrae agree with ROM 65537 in the position of the neural spine completely anterior to the postzygapophysis in lateral view (CMN 50790; FSAC-KK-5019, Smyth et al. 2020:fig. 2); and in having a longer separation (compared to C3 and C4) on the posterodorsal surface of the postzygapophysis in posterior view between the base of the epipophysis and the posterior margin of the postzygapophyseal facet (CMN 50790, Fig. 5B). They differ from ROM 65537 in that the epipophysis is directed more posteriorly, and overhangs the posterior margin of the postzygapophysis. Vertebrae identified by Smyth et al. (2020) as C6 differ from ROM 65537 in having a more elongated centrum, the absence of a hypapophyseal tuberosity, the presence of a ventral keel, and the presence of a posteroventral triangular platform. Although Smyth et al. (2020) identified ontogenetic variation in the development of the keel and the posteroventral triangular platform, these structures were considered inversely correlated, such that a weaker keel is associated with a stronger posteroventral triangular platform.
The absence of both features in ROM 65537 is thus outside the range of variation expected for C6. The positions of the parapophysis and the central pneumatic foramen in CMN 50791 (Evers et al. 2015:fig. 1) are similar to ROM 65537. On the neural arch, the form of the prezygodiapophyseal and posterior laminae of the transvere process in the $\mathrm{C} 6$ vertebrae agree with ROM 65537. The prezygapophyses of the C6 vertebrae are similar to ROM 65537 in being large and projecting far beyond the anterior end of the centrum, but have a smaller angle of divergence in dorsal view. The C6 vertebrae also have the position of the neural spine completely anterior to the postzygapophysis in lateral view, though with a slightly greater separation from the postzygapophysis than in ROM 65537; and a relatively long separation on the posterodorsal surface of the postzygapophysis between the base of the epipophysis and the posterior margin of the postzygapophyseal facet. Although the epipophysis is reduced and has minimal overhang of the posterior end of the postzygapophysis, it remains posteriorly directed, unlike the more vertical epipophysis in ROM 65537.

No complete spinosaurid cervical from the Bahariya Formation or Kem Kem Group identified as C7 has been described, and the anatomy of this position in Spinosaurus is currently characterized mainly on the basis of centra (Smyth et al. 2020), although a partial neural arch is preserved in the possible C7 of FSAC-KK 11888 (Ibrahim et al. 2020a:fig. 130A). These vertebrae differ from ROM 65537 in most of the same centrum characters as the C6 centra, including the presence of a keel and a posteroventral triangular platform.

Posterior cervicals referred to Spinosaurus, beginning with those identified as C8, strongly differ from ROM 65537 in numerous features including dorsal (rather than ventral) flexion of the anterior articular surface; the presence of a median tuberosity on the anterior articular surface; a prominent ventral keel and posteroventral triangular platform; a reniform posterior articular surface; an anteroposteriorly short neural arch with a reduced, spike-like neural spine; the absence of interpostzygapophyseal laminae; and the absence of epipophyses (McFeeters et al. 2013; Evers et al. 2015; Smyth et al. 2020). A hypapophysis is present on the last cervical, C10 (BSPG 2006 I 53, Evers et al. 2015:fig. 7). However, this occurs in combination with a prominent ventral keel, and does not have the low, rounded, moundlike form seen in ROM 65537.

Ibrahim et al. (2020a:fig. 126), in a figure caption, referred to CMN 41768 and CMN 50791 as "mid cervical" vertebrae, and to CMN 50790 and MPDM 33 as "posterior cervical" vertebrae of Spinosaurus. This is contrary to other work identifying CMN 50790 as representing an axial position anterior to CMN 50791 (Russell 1996; Smyth et al. 2020). The identification of CMN 50790 by 
Ibrahim et al. (2020a) as representing a "posterior cervical" position would potentially undermine their proposed referral of all Kem Kem Group spinosaurid material to Spinosaurus aegyptiacus, because it substantially differs from all other specimens that have been interpreted as belonging to the region posterior to CMN 50791 (Evers et al. 2015; Smyth et al. 2020). The new full skeleton reconstruction of Spinosaurus aegyptiacus presented by Ibrahim et al. (2020b:fig. 1G) also differs from that of Smyth et al. (2020:fig. 14) in having tall, anteroposteriorly elongate neural spines on C7-C10, and tall, anteriorly inclined neural spines on D1-D3. However, since Ibrahim et al. (2020a) did not discuss the axial positions of these specimens, and were firm in their argument for a single species of Spinosauridae in the Kem Kem Group, these inconsistencies may have arisen in error, and could be considered supplanted by the more rigorously defended identifications and reconstruction of Smyth et al. (2020). The otherwise undescribed specimen MPDM 33 (Ibrahim et al. 2020a:fig. 126J, K) does not appear to have any major anatomical differences from CMN 50791, which is assigned to C6 (Evers et al. 2015; Smyth et al. 2020), and could belong to the same axial position as it.

\section{COMPARISONS TO OTHER SPINOSAURIDS}

Associated mid-cervical vertebrae are known for the spinosaurids Baryonyx walkeri (Charig and Milner 1997; Evers et al. 2015), Suchomimus tenerensis (Ibrahim et al. 2020a), and Ichthyovenator laosensis (Allain 2014), although the material has not been figured for the last taxon. The mid-cervical vertebrae of Baryonyx and Suchomimus differ from ROM 65537 and all other spinosaurid mid-cervicals from the Kem Kem Group in the possession of a relatively well-developed postzygodiapophyseal lamina (Charig and Milner 1997:fig. 20C-E; Ibrahim et al. 2020a:fig. 130B). Both have mid-cervical epipophyses that overhang the postzygapophyses posteriorly, unlike ROM 65537. The mid-cervical centra of Baryonyx have reniform articular surfaces that are extremely wide compared to their height, less closely resembling ROM 65537 than they do the articular surfaces of more posterior cervical centra from the Kem Kem Group. Suchomimus, conversely, differs from ROM 65537 in reportedly having no cervical centra with posterior articular surfaces that are wider than high (Ibrahim et al. 2020a). None of these taxa have been described as having a mid-cervical centrum with a hypapophyseal tuberosity.

The type material of the spinosaurid Vallibonavenatrix cani includes a partial vertebra, MSMCa-12, identified as a "mid-cervical" by Malafaia et al. (2020). Unusually for a mid-cervical, this specimen has a robust hypapophysis, but unlike in ROM 65537 the hypapophysis in MSMCa-12 is triangular, with a pointed posterior end that is connected to a deep ventral keel. The vertebra MSMCa-12 further differs from ROM 65537 in having divergent anterior and posterior centrodiapophyseal laminae (Malafaia et al. 2020: Fig. 2E), rather than an undivided single centrodiapophyseal lamina. However, Malafaia et al. (2020) did not explain their reasoning for labeling MSMCa-12 a "mid-cervical," and its true axial position may have been more posterior. A complete mid-cervical vertebra from a different locality in the Arcillas de Morella Formation, referred to Spinosauridae indet., has very long, posteriorly overhanging epipophyses, and only a low ventral keel on the centrum (Malafaia et al. 2018).

\section{TAXONOMIC IMPLICATIONS}

The above comparisons demonstrate that ROM 65537 , although exhibiting a diagnostically "mid-cervical" morphology, does not fit comfortably into any of the currently recognized axial positions of Spinosaurus aegyptiacus (sensu Smith et al. 2020), or any other known spinosaurid taxon. The proportions of the centrum suggest that ROM 65537 could represent $\mathrm{C} 4$, while features of the neural arch are more reminiscent of $\mathrm{C} 5$ and $\mathrm{C} 6$, which typically have more elongated centra (Fig. 4). The hypothesis that ROM 65537 could be a chimeric specimen, assembled from the isolated centrum of one vertebra and the isolated neural arch of another, is refuted by the unbroken fit of the two components along the preserved neurocentral suture line. Additionally, features such as the hypapophyseal tuberosity and the dorsally directed epipophysis have not been documented in other specimens of the mid-cervical positions that ROM 65537 most closely resembles. It is possible that Spinosaurus aegyptiacus possessed more than the widely conserved plesiomorphic theropod count of 10 cervicals, and ROM 65537 fits between two previously recognized axial positions. However, this is not considered especially likely, since its morphology is not directly intermediate between any two other axial positions. It is also unlikely that the differences between ROM 65537 and the other specimens can be completely explained by ontogeny, because it is similar in size to other large spinosaurid mid-cervicals from the Kem Kem Group (BSPG 2006 I 57, CMN 50790), and other specimens assigned to the same axial position show a more consistent morphology across a much greater size range.

Does ROM 65537 indicate the presence of a second spinosaurid taxon in the Kem Kem Group? A foreseeable objection to this hypothesis is that ROM 65537 is an isolated anomaly against a large body of evidence otherwise compatible with a single spinosaurid taxon in the Kem Kem Group, and might thus be more parsimoni- 
McFeeters - Vertebral morphotype of Spinosauridae

ously explained by invoking individual variation. Smyth et al. (2020) made this argument in their discussion of an unusual partial quadrate, previously interpreted as evidence of a second spinosaurid in the Kem Kem Group by Hendrickx et al. (2016), although Ibrahim et al. (2020a) were not confident that this quadrate could be identified as spinosaurid. Smyth et al. (2020) considered the individual variation in Spinosaurus aegyptiacus cervical vertebrae comparable to that documented in those of Tyrannosaurus rex (Carpenter 1990), though Carpenter (1990) mentioned only robustness and neural spine shape for non-pathological variation within that species. Carr (2020), however, found the presence of a hypapophyis on cervical vertebrae of $T$. rex to vary ontogenetically, appearing only in the later stages of adulthood. This trend has not yet been established for mid-cervicals of Spinosaurus (Smyth et al. 2020), but if present could potentially explain the hypapophyseal tuberosity of ROM 65537.

Individual variation can often be challenging to separate from taxonomic variation in the fossil record, and these phenomena are related in that individual variation is a prerequisite for natural selection to occur. Ibrahim et al. (2020a) estimated that the Kem Kem Group was deposited over a period of approximately 3.5 to 4.5 million years. While they considered this duration to be fairly short, and consistent with a uniform vertebrate fauna, it is comparable to the span of time over which anagenetic species turnover has been identified in the large theropod Daspletosaurus (Carr et al. 2017). Species having such a relationship might be separated by only a small number of morphological characters, and have many elements of the skeleton that do not diagnostically differ. Additionally, a morphological species transition occurring near the beginning or end of the depositional history could cause fossils of one species to be far less abundant in a unit than the other, even if the species were ecologically similar. In such a scenario, defendable taxonomic splitting could be obscured if anomalies are discounted. Unfortunately, it is currently equivocal whether or not this was the case with spinosaurids in the Kem Kem Group, due to the lack of detailed stratigraphic information for many specimens including ROM 65537.

Smyth et al. (2020:p. 15) expressed doubt that the functional morphology of the neck would greatly differ between two closely related spinosaurid taxa in the same unit, predicting in particular that a ventral triangular platform on $\mathrm{C} 6$ and more posterior cervical vertebrae would be necessarily conserved as "an essential plesiomorphic adaptation within Spinosaurinae." However, they did retain the morphology of the posteroventral triangular platform as an autapomorphy in their diagnosis of Spinosaurus aegyptiacus, recognizing it as diagnostically distinct from potentially homologous precursor states in other spinosaurids such as Ichthyovenator (Evers et al. 2015) and Suchomimus (Ibrahim et al. 2020a). Various other characters of spinosaurid cervicals, despite their apparent functional importance and high variation throughout the cervical series of a single individual or taxon, also exhibit potentially diagnostic taxonomic variation in the details of these character progressions. The composite neck of Spinosaurus aegyptiacus reconstructed by Smyth et al. (2020) resembles Baryonyx in the trend of the cervical centra becoming proportionately more elongate from $\mathrm{C} 4$ to $\mathrm{C} 5$ to $\mathrm{C} 6$, followed by a reversal of this trend resulting in a much more compact centrum by C9 (Charig and Milner 1997; Evers et al. 2015). This trend also remains similar when comparing the absolute lengths of the centra in Baryonyx, rather than their proportions (Charig and Milner 1997:tab. 1). In contrast, Ibrahim et al. (2020a) noted that in a nearly complete neck of Suchomimus, C4-C6 are all fairly similar in length, and may not be reliably distinguishable on the basis of centrum proportions. $\mathrm{C} 7-\mathrm{C} 9$, rather than being proportionately shorter than $\mathrm{C} 6$, are the most elongate in the cervical series (Ibrahim et al. 2020a:fig. 130). Considering the posterior reduction of the epipophyses, their reduction is more extreme in various specimens identified as C6 of Spinosaurus aegyptiacus (Smyth et al. 2020:fig. 7) than in the equivalent elements of Baryonyx (Evers et al. 2015:fig. 26C) and Suchomimus (Ibrahim et al. 2020a:fig. 130B). The practical absence of epipophyses occurs on spinosaurid cervicals from the Kem Kem Group assigned to positions at least as anterior as C8 (BSPG 2006 I 56, Evers et al. 2015:fig 5), with the neural arch of C7 still incompletely represented (Smyth et al. 2020). A similar absence of epipophyses occurs in Baryonyx at least as anteriorly as C9 (Charig and Milner 1997:fig. 22A). However, epipophyses are still present on C9 of Ichthyovenator (Evers et al. 2015), and persist as far posteriorly as D3 in Suchomimus (Ibrahim et al. 2020a). The interpostzygapophyseal laminae also are absent as anteriorly as C8 in the Kem Kem Group material (Evers et al. 2015), and in posterior cervicals of Ichthyovenator (Evers et al. 2015), but are not lost at all through the cervicodorsal transition in Baryonyx (Charig and Milner 1997:fig. 22). This large amount of of variation suggests that rather than being strongly constrained to a common adaptive morphology, the necks of spinosaurids may have been subject to relatively high rates of character change and homoplasy throughout their evolution. As our knowledge of the clade becomes more complete, new spinosaurid taxa may be distinguishable by a unique "mosaic" distribution of cervical characters related to axial position, in addition to any potential autapomorphies. If at least two spinosaurid taxa occur in the Kem Kem Group, distinguishable by their mid-cervical vertebral morphology, then the presently available data may be 
insufficient to confidently assign many isolated specimens, including other vertebrae, to one taxon or the other (Chiarenza and Cau 2016). The composite series reconstructed by Smyth et al. (2020), though appearing to be broadly correct in its assessment of axial positions, could possibly incorporate material from multiple taxa. It is also uncertain which names would apply to the taxa in this situation, given the non-overlapping nature of the type specimens. If ROM 65537 represents axial position C5, it could potentially have no precise overlap with either the original holotype or neotype of Spinosaurus aegyptiacus (interpreted as preserving axial positions $\mathrm{C} 3$ and $\mathrm{C} 4$, and $\mathrm{C} 2$ and ? $\mathrm{C} 7$, respectively; Smyth et al. 2020), or with the holotypes of either Spinosaurus maroccanus (C6) or Sigilmassasaurus brevicollis (D1). This problem of identity may not be conclusively resolved until the discovery of new material that includes a more complete, associated cervical series. ROM 65537 is provisionally referred here to Spinosauridae indet.

\section{CONCLUSIONS}

The specimen ROM 65537 represents a new spinosaurid mid-cervical morphotype from the Kem Kem Group of Morocco, with a unique combination of characters not previously documented in a single element. The occurrence of a new spinosaurid mid-cervical morphotype in the Kem Kem Group may be unexpected if one accepts the referral of all spinosaurid material from this unit to a single species, Spinosaurus aegyptiacus, for which representative specimens of all expected post-atlas cervical vertebrae have been previously identified (Ibrahim et al. 2020a; Smyth et al. 2020). The morphology of ROM 65537 could be interpreted as representing a greater degree of intraspecific variation in the cervicals of Spinosaurus aegyptiacus than previously recognized, or alternatively, may provide new evidence for the occurrence of two spinosaurid taxa in the Kem Kem Group. The discovery of more material will hopefully allow for future testing of the axial position and taxonomic identity of this unusual specimen.

\section{ACKNOWLEDGEMENTS}

I would first like to thank David Evans for bringing ROM 65537 to my attention and encouraging this research. I also thank Kevin Seymour for assisting with information on the specimen, Margaret Currie and Kieran Shepherd for access to spinosaurid material at the CMN, David Hone and Nizar Ibrahim for their helpful reviews, and Michael Ryan for earlier discussion of the manuscript.

\section{LITERATURE CITED}

Arden, T.M.S., C.G. Klein, S. Zouhri, and N.R. Longrich. 2019. Aquatic adaptation in the skull of carnivorous dinosaurs (Theropoda: Spinosauridae) and the evolution of aquatic habits in spinosaurids. Cretaceous Research 93:275-284.

Allain, R. New material of the theropod Ichthyovenator from Ban Kalum type locality (Laos): implications for the synonymy of Spinosaurus and Sigilmassasaurus and the phylogeny of Spinosauridae. Journal of Vertebrate Paleontology, Program and Abstracts, 2014:78.

Beevor, T., A. Quigley, R.E. Smith, R.S.H. Smyth, N. Ibrahim, S. Zouhri, and D.M. Martill. 2021. Taphonomic evidence supports an aquatic lifestyle for Spinosaurus. Cretaceous Research 117:13pp. DOI: 10.1016/j.cretres.2020.104627

Benson, R.B.J., R.J. Butler, M.T. Carrano, and P.M. O'Connor. 2012. Air-filled postcranial bones in theropod dinosaurs: physiological implications and the 'reptile'-bird transition. Biological Reviews 87:168-193.

Buffetaut, E., 1989. New remains of the enigmatic dinosaur Spinosaurus from the Cretaceous of Morocco and the affinities between Spinosaurus and Baryonyx. Neues Jahrbuch für Geologie und Paläontologie, Monatshefte 1989:79-87.

Carpenter, K. 1990. Variation in Tyrannosaurus rex; pp. 141-145 in K. Carpenter, and P.J. Currie (eds.), Dinosaur Systematics: Approaches and Perspectives. Cambridge University Press, Cambridge.

Carr, T.D. 2020. A high resolution growth series of Tyrannosaurus rex obtained from multiple lines of evidence. PeerJ 8: e9192, 1-103.

Carr, T.D., D.J. Varricchio, J.C. Sedlmayr, E.M. Roberts, and J.R. Moore. 2017. A new tyrannosaur with evidence for anagenesis and a crocodile-like facial sensory system. Scientific Reports 7(44942):1-11.

Charig, A. J., and A.C. Milner. 1997. Baryonyx walkeri, a fish-eating dinosaur from the Wealden of Surrey. Bulletin of the Natural History Museum, Geology Series 53:11-70.

Chiarenza, A.A., and A. Cau. 2016. A large abelisaurid (Dinosauria, Theropoda) from Morocco and comments on the Cenomanian theropods from North Africa. PeerJ 4:e1754, 1-24.

Evers, S.W., O.W.M. Rauhut, A.C. Milner, B. McFeeters, and R. Allain. 2015. A reappraisal of the morphology and systematic position of the theropod dinosaur Sigilmassasaurus from the "middle" Cretaceous of Morocco. PeerJ 3: e1323, 1-101.

Gimsa, J., R. Sleigh, and U. Gimsa. 2016. The riddle of Spinosaurus aegyptiacus' dorsal sail. Geological Magazine 153: 544-547.

Heckeberg, N.S., and O.W.M. Rauhut. Histology of spinosaurid teeth from the Albian-Cenomanian of Morocco: implications for tooth replacement and ecology. Palaeontlogia Electronica 23(a48):1-18. 
Henderson, D.M. 2018. A buoyancy, balance and stability challenge to the hypothesis of a semi-aquatic Spinosaurus Stromer, 1915 (Dinosauria: Theropoda). PeerJ 6:e5409, 1-29.

Hendrickx, C., O. Mateus, and E. Buffetaut. 2016. Morphofunctional analysis of the quadrate of Spinosauridae (Dinosauria: Theropoda) and the presence of Spinosaurus and a second spinosaurine taxon in the Cenomanian of North Africa. PLoS One 11:e144695, 1-49.

Hone, D.W.E., and T.R. Holtz, Jr. 2017. A century of spinosaurs - a review and revision of the Spinosauridae with comments on their ecology. Acta Geologica Sinica (English Edition) 91:1120-1132.

Hone, D.W.E. and T.R. Holtz, Jr. (In press). Comment on: Aquatic adaptation in the skull of carnivorous dinosaurs (Theropoda: Spinosauridae) and the evolution of aquatic habits in spinosaurids. Cretaceous Research.

Ibrahim, N., P.C. Sereno, C. Dal Sasso, S. Maganuco, M. Fabbri, D.M. Martill, S. Zouhri, N. Myhrvold, and D.A. Iurino. 2014. Semiaquatic adaptations in a giant predatory dinosaur. Science 345:613-1616.

Ibrahim, N., P.C. Sereno, D.J. Varricchio, D.M. Martill, D.B. Dutheil, D.M. Unwin, L. Baidder, H.C.E. Larsson, S. Zouhri, and A. Kaoukaya. 2020a. Geology and paleontology of the Upper Cretaceous Kem Kem Group of eastern Morocco. ZooKeys 928:1-216.

Ibrahim, N., S. Maganuco, C. Dal Sasso, M. Fabbri, M. Auditore, G. Bindellini, D.M. Martill, S. Zouhri, D.A. Mattarelli, D.M. Unwin, J. Wiemann, D. Bonadonna, A. Amane, J. Jakubczak, U. Joger, G.V. Lauder, and S.E. Pierce. 2020 b. Tail-propelled aquatic locomotion in a theropod dinosaur. Nature 581:67-70.

Lakin, R.J., and N.R. Longrich. 2019. Juvenile spinosaurs (Theropoda: Spinosauridae) from the middle Cretaceous of Morocco and implications for spinosaur ecology. Cretaceous Research 93:129-142.

Maganuco, S., and C. Dal Sasso. 2018. The smallest biggest theropod dinosaur: a tiny pedal ungual of a juvenile Spinosaurus from the Cretaceous of Morocco. PeerJ 6: e4785, 1-10.
Malafaia, E., J.M. Gasulla, F. Escaso, I. Narváez, J.L. Sanz, and F. Ortega. 2018. New spinosaurid (Theropoda, Megalosauroidea) remains from the Arcillas de Morella Formation (upper Barremian) of Morella, Spain. Cretaceous Research 92:174-183.

Malafaia, E., J.M. Gasulla, F. Escaso, I. Narváez, J.L. Sanz, and F. Ortega. 2020. A new spinosaurid theropod (Dinosauria: Megalosauroidea) from the upper Barremian of Vallibona, Spain: Implications for spinosaurid diversity in the Early Cretaceous of the Iberian Peninsula. Cretaceous Research 106:104221, 1-17.

McFeeters, B., M.J. Ryan, S. Hinic-Frlog, and C. SchröderAdams. 2013. A reevaluation of Sigilmassasaurus brevicollis (Dinosauria) from the Cretaceous of Morocco. Canadian Journal of Earth Sciences 50:636-649.

Russell, D.A. 1996. Isolated dinosaur bones from the Middle Cretaceous of the Tafilalt, Morocco. Bulletin du Muséum national d'Histoire naturelle, Paris, 4e série 18:349-402.

Sereno, P.C., A.L. Beck, D.B. Dutheil, B. Gado, H.C.E. Larsson, G.H. Lyon, J.D. Marcot, O.W.M. Rauhut, R.W. Sadleir, C.A. Sidor, D.D. Varricchio, G.P. Wilson, and J.A. Wilson. 1998. A long-snouted predatory dinosaur from Africa and the evolution of spinosaurids. Science 282:1298-1302.

Smyth, R.S.H., N. Ibrahim, and D.M. Martill. 2020. Sigilmassasaurus is Spinosaurus: a reappraisal of African spinosaurines. Cretaceous Research 114:104520, 1-28.

Stromer, E. 1915. Ergebnisse der Forschungsreisen Prof. Stromers in den Wüsten Agyptens. II Wirbeltier-Reste der BaharîjeStufe (unterstes Cenoman). 3. Das Original des Theropoden Spinosaurus aegyptiacus nov. gen., nov. spec. Abhandlungen der Königlich Bayerischen Akademie der Wissenschaften. Mathematisch-physikalische Klasse 28:1-32.

Wilson, J.A. 1999. A nomenclature for vertebral laminae in sauropods and other saurischian dinosaurs. Journal of Vertebrate Paleontology 19:639-653.

Wilson, J.A., M.D. D’Emic, T. Ikejiri, E.M. Moacdieh, and J.A. Whitlock. 2011. A nomenclature for vertebral fossae in sauropods and other saurischian dinosaurs. PLoS One 6: e17114, 1-19. 